\section{Periodic Classification of Elements}

A MODERN table of chemical elements must show electronic configurations as well as the variation in the propertios of the elements with atomic number. It should also include the lanthanide and actinide series of elements. The trend has been to lengthen the table (for example in the conventional long form and in Simmons' Table ${ }^{1}$ ) and so abolish the sub-group system. In the Table shown herewith the rectangular form has been preserved and a three sub-group system introduced.

Each period corresponds to building up on an inert or pseudo-inert gas structure. They are named according to the orbital which is being filled from group 3 onwards in that period. The basic electronic configuration is shown on the left of each period. The $s$ orbitals which are normally filled after the first two groups are shown in brackets. ments. In the latter case periods $4 f$ and $5 d$ would become one period called $5 d$ and the $5 f$ period would be called $6 d$. The simplest form of the table is very little different from Mendeleef's periodic system. Students of chemistry will only ever need to become familiar with one form which can be adapted for use at all stages of advancement. Advocates of the long forms argue that it is confusing to put differing elements together in the same group. They have even more ground for argument now that $(c)$ sub-groups have been added. But confusion should not occur if it is understood that the resemblances between subgroups are rare and occur only between $(a)$ and $(b)$ sub-groups. It has the advantage that the symbols of the elements with which one is mostly concerned, particularly in the early stages, are more widely spaced and are easier to see.

Mendeleef's classification was an outstanding achievement. Its usefulness has established it in

Table 1. Mendeleef's Table adapted to Show Electronic Configuration

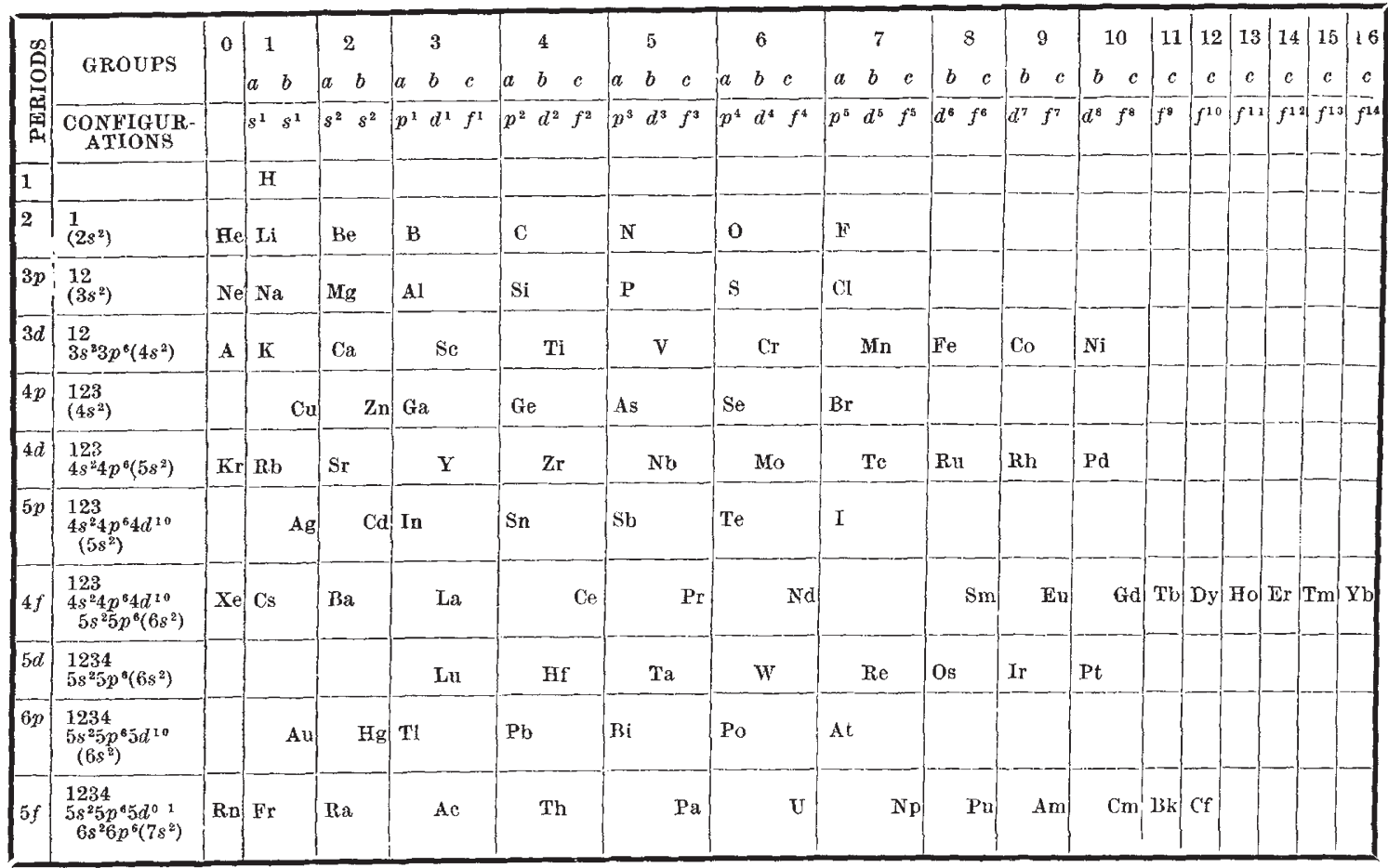

There are 17 groups; the number of each showing the number of electrons in elements of that group in excess of the inert or pseudo-inert gas structures. The sub-groups show which orbitals the electrons might, and in most cases, normally occupy. It is apparent that $(a)$ are representative, (b) transition and (c) inner transition elements. No simple system will show exactly what are believed to be the most stable electronic arrangements. Discrepancies occur among several transition and rare earth elements. However, the table gives a truer picture than Simmons's of the orbitals of the group Ib metals and of actinium and thorium.

It is felt to be important that the form of established Tables should be adhered to as closely as possible and that elements which are commonly referred to as belonging for example to groups IV $a$ or $\mathrm{V} b$ should be found in similar positions in the new Table. The Table, of course, can be drawn without the electronic configurations and/or without the lanthanide ele- chemistry and most inorganic text-books are written around it. It would seem better, therefore, when this is feasible, to adapt it to suit modern requirements, rather than to experiment with new forms.

\section{Balliol College,}

A. A. Clifford

Oxford.

' Simmons, L. M., J. Chem. Educ., 24, 588 (1947) ; 25, 658 (1948).

\section{Redox Properties of some Dipyridyl Quaternary Salts}

IN connexion with an investigation into the mode of action of certain diquaternary salts of $2,2^{\prime}-, 2,4^{\prime}$, and 4,4'-dipyridyl as herbicides ${ }^{\prime}$ the behaviour of a number of dipyridyl diquaternary salts on reduction has been examined.

The complex behaviour of the diquaternary salts of $4,4^{\prime}$-dipyridyl (I) on reduction has long been known, and in 1933 Michaelis and Hill ${ }^{2,3}$, showed that 